\title{
Unilateral Frosted Branch Angiitis Treated with Intravitreal Dexamethasone Implant
}

\author{
Nishikant Borse*, Veena Borse \\ Insight Eye Clinic, Mumbai, India \\ Email address: \\ nishikantborse@yahoo.com (N. Borse), docveenaborse@gmail.com (V. Borse) \\ ${ }^{*}$ Corresponding author
}

To cite this article:

Nishikant Borse, Veena Borse. Unilateral Frosted Branch Angiitis Treated with Intravitreal Dexamethasone Implant. International Journal of Ophthalmology \& Visual Science. Vol. 5, No. 3, 2020, pp. 75-79. doi: 10.11648/j.ijovs.20200503.12

Received: July 17, 2020; Accepted: August 24, 2020; Published: September 24, 2020

\begin{abstract}
Frosted branch angiitis (FBA), a relatively rare condition, is a retinal perivasculitis with severe retinal vessel sheathing resembling the frosted branches of a tree. It can be a Primary Idiopathic FBA or Secondary FBA when associated with systemic conditions like viral infections, sarcoid etc. Primary frosted branch angiitis causes characteristic florid, translucent retinal perivascular sheathing of both arterioles and venules in association with variable uveitis, retinal edema and visual loss. Additional retinal findings may include intraretinal haemorrhages, hard exudates, and serous exudative detachments of the macula and periphery. It is unclear whether retinal frosted branch angiitis is a distinct clinical syndrome or a clinical sign increasingly recognised in a number of inflammatory conditions. Hence, retinal frosted branch angiitis may be more of a sign than a separate disease. Treatment is usually with corticosteroids and normally leads to a good visual recovery. We report a case of primary unilateral frosted branch angiitis with large areas of capillary drop out and severe macular edema. It was successfully managed with intravitreal injection of Dexamethasone Implant (Ozurdex ${ }^{\mathrm{TM}}$ ) in conjunction with a very short course of systemic steroids. To our knowledge, this is the first documented case of primary unilateral FBA treated with systemic steroids and an intravitreal dexamethasone implant.
\end{abstract}

Keywords: Frosted Branch Angiitis, Unilateral, Dexamethasone Implant

\section{Introduction}

Frosted branch angiitis (FBA), is a retinal perivasculitis with severe retinal vessel sheathing resembling the frosted branches of a tree $[1,2]$.

Kleiner [3] proposed that the entity can be divided into primary idiopathic and secondary FBA. The known causes of secondary FBA are viral infections, sarcoidosis, multiple sclerosis, toxoplasmosis, syphilis, Behcet's disease, lymphoma, and leukaemia [2, 3].

This condition is typically bilateral, but unilateral cases (28\%) have been reported.

Unilateral FBA is rare. $[4,5]$. There is also a case report of unilateral FBA associated with vitreous haemorrhage. [6].

In this report, we present a rare case of primary unilateral FBA successfully managed with short term oral steroids and an intravitreal injection of Ozurdex ${ }^{\mathrm{TM}}$.

\section{Case Report}

A 44-year-old man presented with blurred vision in the left eye, since 5 days.

There was no other significant ophthalmic or medical history.

On examination, visual acuities were $20 / 20$ in the right eye and 20/200 in the left eye on the Snellen's Visual Acuity Chart. There was a mild relative afferent pupillary defect in the left eye.

On slit lamp examination, there was a mild circumcorneal congestion with $1+$ anterior chamber cells indicating a mild anterior uveitis. Intraocular pressures were $16 \mathrm{mmHg}$ in both the eyes with applanation tonometry. Fundus examination (Figure 1) revealed retinal vasculitis with prominent sheathing affecting the veins as well as the arteries of the superotemporal quadrant extending to the periphery in the left eye. These veins were also dilated and tortuous due to a resultant inflammatory venous occlusion. There were multiple retinal haemorrhages extending to the periphery (Figure 2). 


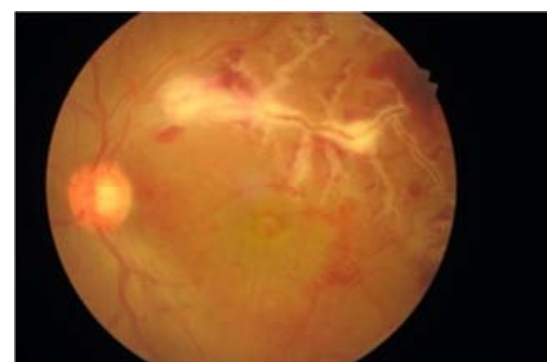

Figure 1. Frosted Branch Angiitis involving the superotemporal blood vessels.

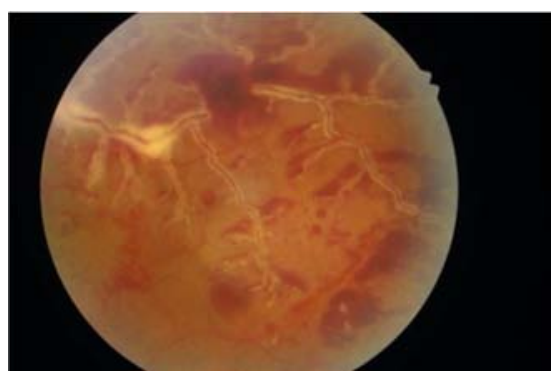

Figure 2. FBA extending to the temporal periphery along with retinal haemorrhages.

The macula showed severe macular edema. and scattered small retinal haemorrhages.

The right eye had a normal retina.

He was subjected to ophthalmological as well as systemic investigations.

The blood count and differential cell count were normal, as were the erythrocyte sedimentation rate, $\mathrm{C}$-reactive protein and chest X-ray.

The HIV antibody test \& serology for cytomegalovirus, herpes simplex, varicella zoster and Epstein-Barr viruses, rubella, and syphilis and Toxoplasma gondii were negative.

Fluorescein angiography (FA) showed a delayed retinalartery-to-vein transit time.

The early FA images showed a large area of capillary non perfusion in the superotemporal area extending to the periphery. The superotemporal vein was dilated and tortuous with a venous beading like appearance. (Figure 3 ).

There was a total branch vein occlusion involving the macular branch of the superotemporal vein. The later phases showed a diffuse leakage from the inflamed blood vessels, involving the macular area. (Figure 4). There was a late diffuse staining and leakage of the affected vessels suggestive of diffuse retinal periphlebitis (Figure 5)

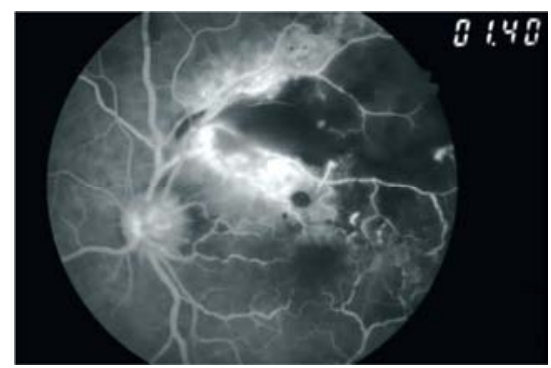

Figure 3. Early Fluorescein Angiogram picture showing vasculitis and large areas of capillary dropouts in the superotemporal quadrant.

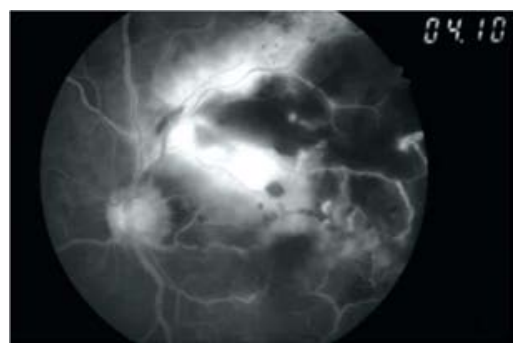

Figure 4. Late phase FA showing leakage of the dye from the inflamed blood vessels into the macular area.

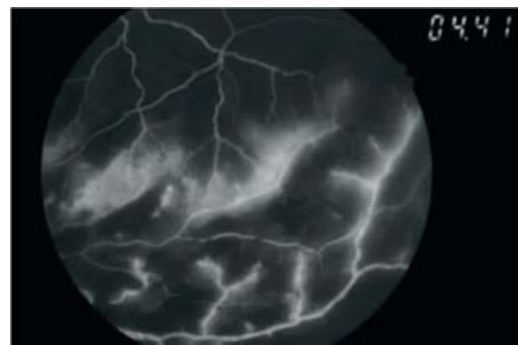

Figure 5. Typical Vascular Staining of the blood vessels due to diffuse retinal periphlebitisin in late phases of FA.

The Spectral Domain Optical Coherence Tomography (SDOCT) showed severe macular edema with a macular thickness of about 700 microns (Figure 6) The edema was subretinal as well as intraretinal mainly involving the outer retina.

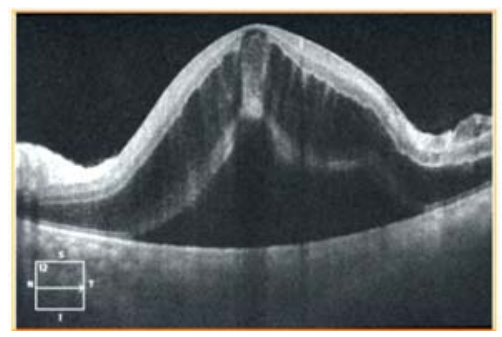

Figure 6. SD-OCT through the fovea of the right eye $(O D)$ showing a large amount of cystoid macular edema and a serous foveal detachment.

Based on these findings a diagnosis of Primary Frosted Branch Angiitis (FBA) was made.

He was treated with oral prednisolone $1 \mathrm{mg} / \mathrm{kg} / \mathrm{day}$, with topical steroid and mydriatic.

Since the macular edema was so severe he was given an intravitreal injection of Ozurdex ${ }^{\mathrm{TM}}$.

The clinical response was rapid.

The visual acuity improved to $20 / 60$ in the left eye within a week

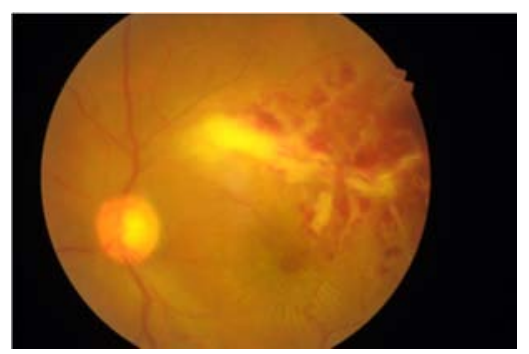

Figure 7. Resolving Vasculitis, Haemorrhages \& Resolving Macular Edema seen within a week of treatment. 
The ocular congestion subsided and there were no signs of anterior uveitis. The retina showed a significantly reduced periphlebitis though the proximal part of the superotemporal retinal vein did show persistent phlebitis. (Figure 7). The macular edema showed near complete resolution by the $6^{\text {th }}$ day, as seen on the SD OCT macula. (Figure 8).

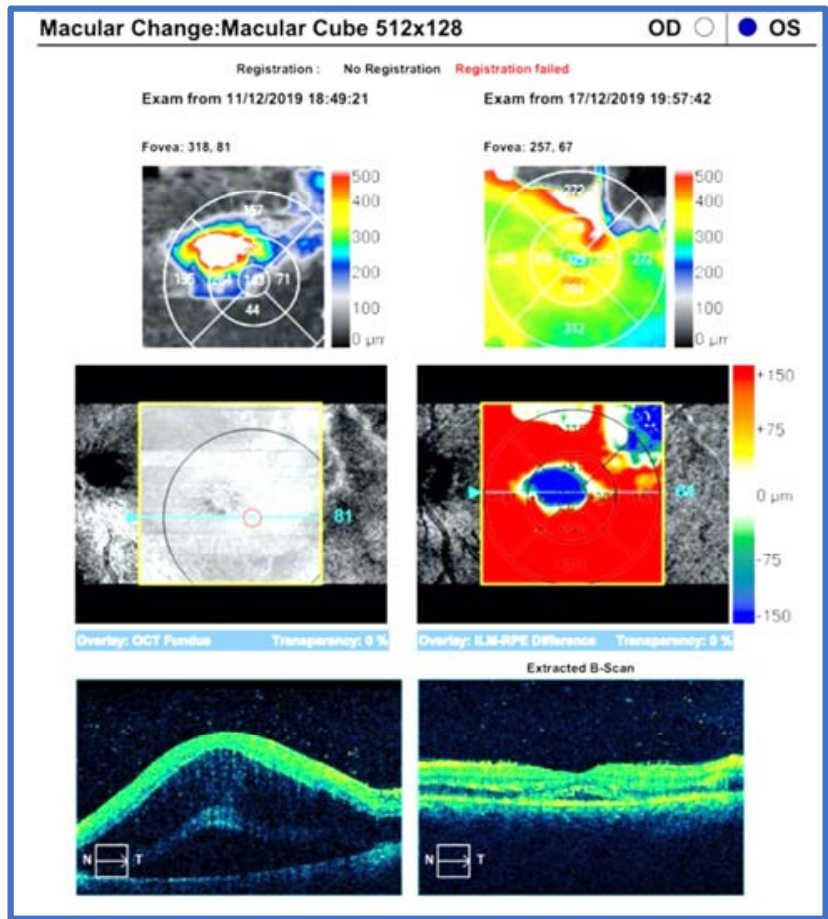

Figure 8. SD OCT - Macular Change Analysis showing the dramatic resolution of Macular edema within a week of onset of treatment.

The systemic steroid treatment was tapered and discontinued within 4 weeks.

After 4 weeks, the peri phlebitis had nearly resolved (Figure 9) and the macular edema had resolved fully. The retinal vessels showed resolution of the FBA. There were persistent retinal haemorrhages extending to the retinal periphery which gradually resolved over 12 weeks of starting the treatment.

The SD OCT done at 4 weeks showed a complete resolution of macular edema. However, there was some macular thinning which may be due to the ischemia caused by the vascular occlusion. (Figure 10) The patient however maintained a visual acuity of $20 / 60$.

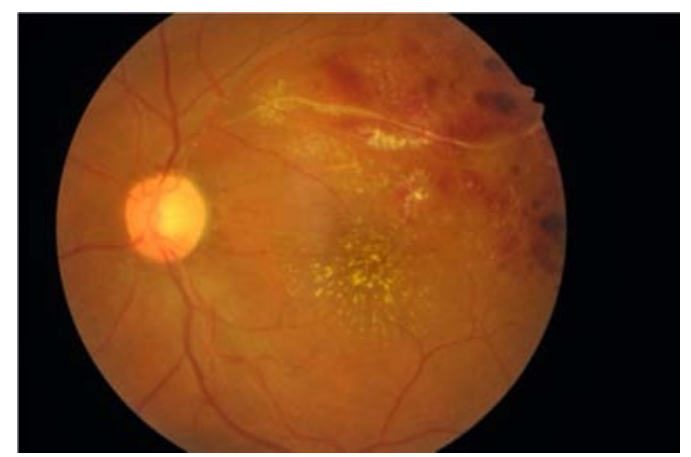

Figure 9. Near Complete Resolution of the Vasculitis at 4 weeks.

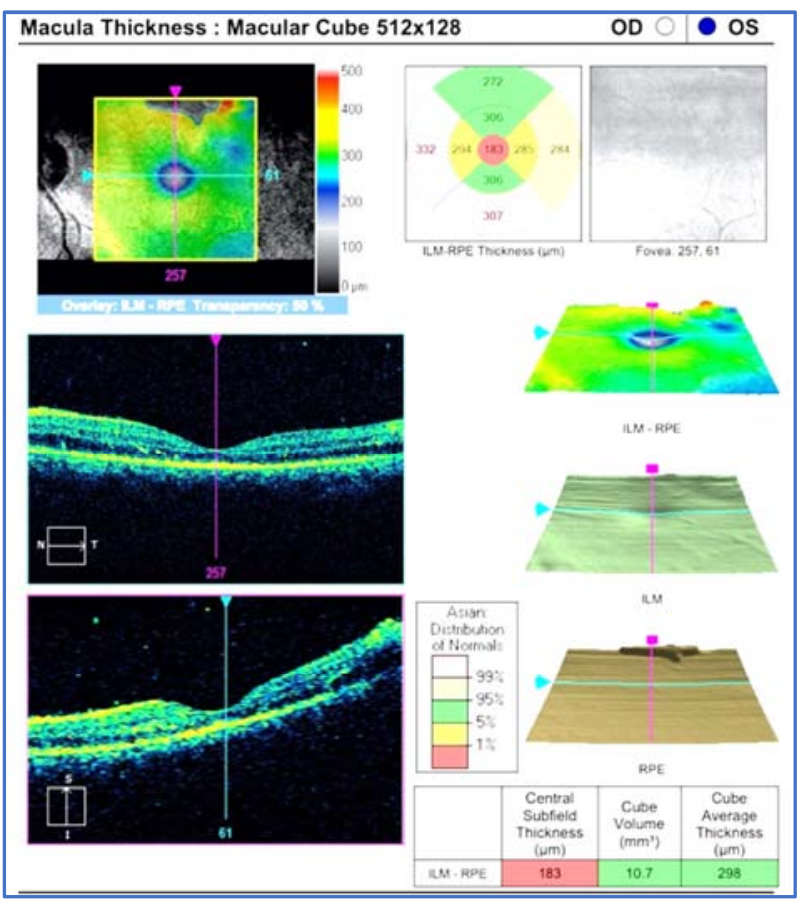

Figure 10. SD OCT macula at 4 weeks. There is complete resolution of the macular edema. There is foveal thinning which may be due to the ischemia associated with the vasculitis.

He was subsequently maintained only on a twice a day dose of topical steroid which was tapered off over the next 4 weeks.

There has been no recurrence even after 6 months of treatment and as of today the patient is not on any medications.

\section{Discussion}

In 1976, Ito [5] reported a 6-year-old Japanese boy with pan uveitis and widespread retinal vasculitis. The florid translucent perivascular exudate inspired the descriptive term 'frosted branch angiitis' recalling imagery of frosted tree branches during an icy winter.

Frosted branch angiitis may be idiopathic or associated with various systemic conditions including SLE [8, 9], Bechet's disease [10], Crohn's disease [11], cytomegalovirus (CMV) retinitis [12], herpes simplex type 2 infection [13], mycobacterium tuberculosis infection [14], T. gondii infection [15], various other viral and bacterial infections [16], aseptic meningitis [17], and blood dyscrasias such as leukaemia [18] and lymphoma [19].

Additional retinal findings may include intraretinal haemorrhages, hard exudates, and serous exudative detachments of the macula and periphery. A proposed mechanism of retinopathy involves immune complex deposition in retinal vessels causing vasculitis [20].

Patients may complain of sudden onset of blurring of vision, central scotomas, floaters, and photopsia and most patients respond to systemic corticosteroid therapy with good recovery of visual acuity. [21].

It is unclear whether retinal frosted branch angiitis is a distinct clinical syndrome or a clinical sign increasingly recognised in a number of inflammatory conditions. [22] 
Hence retinal frosted branch angiitis may be more of a sign than a separate disease.

Our patient presented with acute idiopathic unilateral FBA. Other than being unilateral, the other unique characteristics were a large area of capillary drop out due to an inflammatory vascular occlusion and a very severe macular edema.

It is well known that FBA responds well to systemic steroids. However, the large area of ischemia and the severity of the macular edema prompted us to use an intravitreal dexamethasone implant since we felt an aggressive approach was necessary for rapid resolution of the ocular inflammation, thereby by relieving the resultant vascular occlusion.

Ozurdex ${ }^{\mathrm{TM}}$ is a biodegradable intravitreal dexamethasone implant approved by the United States Food and Drug Administration for treatment of macular edema associated with central retinal vein occlusion (CRVO) and for treatment of non-infectious posterior uveitis. [23-26]. Since macular edema secondary to uveitis is difficult to treat and may persist despite multiple interventions [27], we decided supplement the oral steroid treatment with an Ozurdex ${ }^{\mathrm{TM}}$ implant.

In our case, this supplemental treatment helped a rapid resolution of inflammation as well as the macular edema with in week. The central retinal thickness as seen on the SD OCT showed a rapid reduction within a week of initiating the treatment. This, of course corelated with a significant visual gain. It also facilitated an early tapering off of the systemic steroids thereby preventing most of the oral steroid related adverse effects.

It also helped to have a sustained response and may have prevented a recurrence of the inflammation.

Hence we feel that use of Ozurdex ${ }^{\mathrm{TM}}$ may be a useful tool in rapid resolution of the inflammation and the associated macular edema. This also helps in faster restoration of vision which is sustained due to the longer duration of action of the dexamethasone implant.

To our knowledge there is only one report of use of Ozurdex $^{\mathrm{TM}}$ in FBA.

However, that report by Dong Yoon Kim et al was in a case of FBA Secondary to Granulomatosis with Polyangiitis [28]

To our knowledge, this is the first documented case of primary unilateral FBA treated with systemic steroids and an intravitreal dexamethasone implant.

\section{Conclusion}

Primary FBA is rare. When it is associated with a severe macular edema and also a vascular occlusion secondary to the vasculitis it has to be treated aggressively. Though FBA is known to respond well to systemic corticosteroid treatment, dexamethasone implant can be a useful adjunct in treating FBA.

It helps rapid resolution of inflammation , macular edema and the retinal vasculitis. The sustained action of the implant helps to avoid prolonged oral steroid therapy thereby reducing its systemic side effects.

We therefore feel Ozurdex ${ }^{\mathrm{TM}}$ can be considered as a useful adjunct to oral corticosteroid treatment in management of Primary FBA.

\section{Conflicts of Interest}

All the authors do not have any possible conflicts of interest.

\section{References}

[1] Sandhu RK, Adams T, Sibley C, et al. Granulomatosis with polyangiitis (Gap) presenting with frosted branch angiitis. Retina Cases Brief Rep 2016; 10: 249-51.

[2] Walker S, Iguchi A, Jones NP. Frosted branch angiitis: a review. Eye (Lond) 2004; 18: 527-33.

[3] Kleiner RC. Frosted branch angiitis: clinical syndrome or clinical sign? Retina 1997; 17: 370.

[4] Sugin SL, Henderly DE, Friedman SM, Jampol LM, Doyle JW. Unilateral frosted branch angiitis. Am J Ophthalmol 1991; 111: 682-85.

[5] Seo MS, Woo JM, Jeong SK, Park YG. Recurrent unilateral frosted branch angiitis. Jpn J Ophthalmol 1998; 42: 56-59.

[6] Agrawal S, Agrawal J, Agrawal T P. Unilateral frosted branch angiitis with vitreous haemorrhage. Indian J Ophthalmol2001; 49: 269-70.

[7] Ito Y, Nakano M, Kyu N, Takeuchi M. Frosted branch angiitis in a child. Jpn J Clin Ophthalmol 1976; 30: 797-803.

[8] Hernandez-Da Mota SE, Arellanes-Garcia L, Recillas-Gispert C et al (2011) Lupus relapse presented as frosted branch retinal angiitis: case report. Ocul Immunol Inflamm 19: 367 369.

[9] Quillen DA, Stathopoulos NA, Blankenship GW, Ferriss JA (1997) Lupus associated frosted branch periphlebitis and exudative maculopathy. Retina 17: 449-451.

[10] Kwon SJ, Park DH, Shin JP (2013) Frosted branch angiitis as ocular manifestation of Behcet's disease: unusual case report and literature review. Korean J Ophthalmol 27: 466-469. doi: 10.3341/kjo.2013.27.6.466.

[11] Sykes SO, Horton JC (1997) Steroid-responsive retinal vasculitis with a frosted branch appearance in Crohns disease. Retina 17: 451-454.

[12] Spaide RF, Vitale AT, Toth IR, Oliver JM (1992) Frosted branch angiitis associated with cytomegalovirus retinitis. Am J Ophthalmol 113: 522-528.

[13] Markomichelakis NN, Barampouti F, Zafirakis P et al (1999) Retinal vasculitis with a frosted branch angiitis-like response due to herpes simplex virus type 2. Retina 19: 455-45.

[14] Zhao H, Guan J, Zhou L et al (2012) Frosted branch angiitis in a woman with Mycobacterium tuberculosis infection. Ocul Immunol Inflamm 20: 227-229. doi: $10.3109 / 09273948.2012 .673044$ 
[15] Suzuki T, Onouchi H, Nakagawa $Y$ et al (2010) A case of recurrence of congenital ocular toxoplasmosis with frosted branch angiitis (ocular toxoplasmosis with frosted branch angiitis). Tokai J Exp Clin Med 35: 122-125.

[16] Walker S, Iguchi A, Jones NP (2004) Frosted branch angiitis: a review. Eye18: 527-533. doi: 10.1038/sj.eye.6700712

[17] Matsui Y, Tsukitome H, Uchiyama E et al (2013) Peripheral capillary nonperfusion and full field electroretinographic changes in eyes with frosted branch-like appearance retinal vasculitis. Clin Ophthalmol 7: 137-140. doi: 10.2147/OPTH.S40110

[18] Kim TS, Duker JS, Hedges TR (1994) Retinal angiopathy resembling unilateral frosted branch angiitis in a patient with relapsing acute lymphoblastic leukaemia. Am J Ophthalmol 117: 806-808.

[19] Hua MT, Blaise P, De Leval L, Rakic JM (2009) Frosted branch angiitis with undiagnosed Hodgkin lymphoma. Eur J Ophthalmol 19: 310-313.

[20] Wong R, Cunningham E (2013) Posterior segment findings in SLE. Rev Ophthalmol 20: 41-45.

[21] Wood and Wong Journal of Ophthalmic Inflammation and Infection (2016) 6: 20 DOI 10.1186/s12348-016-0089-9.

[22] Frosted Branch Angiitis: Elisabetta Miserocchi, M. D. Available at: http://www.uveitis.org/docs/dm/frosted_branch_angiitis.pdf.
[23] Robinson MR, Whitcup SM. Pharmacologic and clinical profile of dexamethasone intravitreal implant. Expert Rev Clin Pharmacol. 2012; 5: 629-47. (PubMed) (Google Scholar).

[24] Haller JA, Bandello F, Belfort R, Jr, Blumenkranz MS, Gillies $\mathrm{M}$, Heier J, et al. Randomized, sham-controlled trial of dexamethasone intravitreal implant in patients with macular edema due to retinal vein occlusion. Ophthalmology. 2010; 117: 1134-46.e3. (PubMed) (Google Scholar).

[25] Haller JA, Bandello F, Belfort R, Jr, Blumenkranz MS, Gillies $\mathrm{M}$, Heier $\mathrm{J}$, et al. Dexamethasone intravitreal implant in patients with macular edema related to branch or central retinal vein occlusion twelve-month study results. Ophthalmology. 2011; 118: 2453-60. (PubMed) (Google Scholar).

[26] Lowder C, Belfort R, Jr, Lightman S, Foster CS, Robinson MR, Schiffman RM, et al. Dexamethasone intravitreal implant for noninfectious intermediate or posterior uveitis. Arch Ophthalmol. 2011; 129: 545-53. (PubMed) (Google Scholar).

[27] Karim R, Sykakis E, Lightman S, Fraser-Bell S. Interventions for the treatment of uveitic macular edema: A systematic review and meta-analysis. Clin Ophthalmol. 2013; 7: 110944. (PMC free article) (PubMed) (Google Scholar).

[28] Dong Yoon Kim, Jinho Jeong \& Jin Young Kim; Frosted Branch Angiitis Secondary to Granulomatosis with Polyangiitis Korean J Ophthalmol. 2019 Oct; 33 (5): 485-486. 